carcinoma of the caecum. Carcinoma of the colon is relatively less of a problem in so far as it is accessible to sigmoidoscopy, and so is Crohn's disease in so far as it involves the ileum. But the forms of amoebiasis that mimic these conditions are all detected with considerable success serologically. So also is liver abscess. "Diarrhoea of more than two weeks" duration," if due to amoebiasis, might give an equivocal serological result and is best investigated by competent stool examination, which, of course, should not be omitted in the other conditions also.

The routine diagnostic services provided by the amoebiasis unit when it was under the direction of Air Vice-Marshal Stamm are unabated and continue to be freely available, though the unit now operates with a reduced staff. As a reference centre for serological diagnosis, advice, and assistance with identifi cation of organisms it meets the need. But it is unrealistic to think that a reference centre can undertake the first-line investigation of all the stool specimens from all the patients who might have amoebiasis any more than it could do the same for blood films for malaria. Nor is the examination of fixed stools the ideal, or acceptable as a routine. A lot of this work is going to continue to be done in general microbiology departments. From this point of view one can only plead for a much more thorough training of more pathologists and technician in diagnostic parasitology. At present we offer neither the training, nor a qualification, nor a career prospect in this subject.

As you say, Sir (p 379), continuing publicity is needed, and your leading article therefore is welcome. But your conclusion that the amoebiasis unit has lost its identity by a move from St Giles's Hospital to the Hospital for Tropical Diseases is difficult to follow.

D S RIDLEY

D C WARHURS

Amoebiasis Unit,

Hospital for Tropical Diseases,

London NW1

SIR,-As a retired general practitioner I feel diffident in writing on amoebiasis, but 3 years' recent experience in central north India treating large numbers of cases of diarrhoea in otherwise healthy Europeans has given me thoughts on this subject.

I found that laboratory microscopic diagnosis was not reliable, but the history was usually fairly consistent. This was of abdominal colic with a change of bowel habit -for instance, of large, soft, gaseous stool which tended to float on water in the pan and which tended to occur later in the day than usual. In nearly all cases this was preceded by the onset of anorexia and unusual mental and physical lethargy. Fever was not a particula feature except in more severe cases, when there might be blood and mucus in the stools as well.

The results of treatment with metronidazole, with rest and extra fluids and Electroso tablets (containing sodium and potassium chlorides and sodium bicarbonate) when possible, were so remarkably good that I found that this drug could be reliable as a diagnostic therapeutic tool. I found that many person could not tolerate the usually recommended dose of $400 \mathrm{mg}$ three times a day for five days on account of severe nausea, but half this dos for 10 days was acceptable and equally efficacious. Suitable doses for children also gave very satisfactory results.
I cannot help wondering in view of the remarks in your leading article (5 August, p 379) whether administration of metronidazole might be used as a diagnostic method in obscure cases of bowel or liver disorders when sophisticated techniques such as fluorescent antibody tests are not available and when amoebiasis cannot be excluded. Metronidazole seemed to me to be a safe drug to use.

\section{S G HAMILTON}

Leiston, Suffolk

\section{When and why are babies weaned?}

SIR,-Drs P W Wilkinson and D P Davies should be congratulated for injecting a note of realism and reason into the absurd argumen over when babies should be weaned ( 24 June $p$ 1682). To discourage the introduction of solids before 4-6 months may be a counsel of necessity in many parts of the world but makes nonsense in the UK for the following reasons.

(1) Hunger-It is common knowledge that many babies become ravenously hungry in the third month and cry a great deal-as many as $80 \%$ in Drs Wilkinson and Davies's small sample. Hunger is the commonest cause of so-called "three months" colic." The physiological basis of this excessive crying has yet to be demonstrated, but it may well be related to such factors as gastric emptying and reactive hypoglycaemia. The problem can, of course, be easily countered by feeding the baby every 2-3 hours with milk, an infallible recipe for obesity, which the discouragement of solids is supposed to avoid. Now that an increasing number of babies are fully breast-fed it is already apparen that the frequently breast-fed baby can become ust as fat as his frequently bottle-fed brother. The early introduction of low-carbohydrate solid is both rational and nutritionally correct.

(2) Allergy-While babies do not develop allergy to human milk, it is a fact that sooner or late they are given cows' milk-fewer than $20^{\circ}$ of babies in the UK are still breast-fed after 3 month -and the more they drink the more likely they are to develop symptoms of allergy. An intake of two pints daily (five $8-\mathrm{oz}$ bottles) is roughly equal to an intake of 20 pints daily for an average adult on a weight-for-weight basis. The sheer immensity of the protein challenge is a sure prescription for the development of constan snuffling, repeated wheezy bronchitis, vomiting, transient rashes, and several other common symptoms of this age group. The problem can be avoided or improved by reducing the intake of milk and distributing the protein requirement over more varied range.

(3) Nutrition-It is often said that human milk contains everything the infant needs, but th artificialities of Western diets, climate, and culture may combine to produce less than optimal nutrition. Anaemia is relatively common, and my last case of florid rickets was in a West Indian infant who had been fully breast-fed without any supplements for 10 months. Certainly a diet of cows' milk alone would not be considered balanced diet for an adult and even the modified fortified formulae currently used for babies may have lost trace substances in the process of manufacture. This is well recognised in the case of soya-based and low-lactose products, for which a complex supplement of vitamins, minerals, and trace elements is essential. In my experience the simplest, safest, and least expensive supplemen is a small quantity of a natural food such as egg, chicken, carrot, banana, or apple. It is not sufficiently recognised that as little as two or three teaspoonfuls are sufficient to prevent the development of bizarre deficiency states after several weeks. Recent work in this laboratory suggests that both human milk and the new highly modified milks for babies contain too little sodium for optimal growth; hyponatraemia is common and inhibits normal growth in some babies. The fact that so many babies are evidently dissatisfied on milk alone cannot be ignored.

(4) Habituation-Every baby is a potential breast or bottle addict. Management problems arising from the introduction of other tastes and methods can be considerable if this is postponed until 6 months.

It is necessary not to confuse the introduction of new tastes and methods and the possible supply of trace substances with "weaning," by which is meant the replacement of milk by other foods. The word "solids" itself is a misnomer, referring as it does to strained foods with a water content of nearly $90 \%$ and even liquids. A spoonful or two of a strained food is not necessarily synonymous with taking the baby off milk. Current propaganda has led to the absurd situation of mothers begging nervously for permission to give their babies a little orange juice or sieved carrot or staying away from the clinic for fear of the dose of "strong discouragement" which will be meted out by the health visitor. Fortunately, as Drs Wilkinson and Davies have found, most mothers know when to introduce other foods with the minimum of guidance.

HERBERT BARRIE

Paediatric Research Laboratory,

Charing Cross Hospita!,

\section{Sexual pressures on children}

SIR,-The Reverend J B Metcalfe's letter (12 August, p 499) is morally unsound. The moral law cannot be based on what may be the norm in some societies. The function of the law is not to record what is being done but what should be done. An excellent reference is to be found in Matthew v, 27-30.

There is abundant medical evidence clearly demonstrating the damaging social and health hazards which result from the young indulging in unrestrained sexual activity. There has been an enormous rise in induced abortion in the under-19 age group, 27963 having been recorded in 1977. It is these young people who will have to suffer the consequences of induced abortion such as spontaneous abortions in subsequent pregnancies, premature labours, ectopic pregnancies, and sterility. The increased illegitimacy rate in the 15-19 age group, the upsurge of venereal infection in the young, and the clearly established link between cancer of the cervix, promiscuity, and early age of intercourse should give $\mathrm{Mr}$ Metcalfe all the facts he needs.

The presence of other evils in society such as car accidents and bad housing cannot logically be cited as a reason for propagating additional evils.

\section{J H Scotson}

Timperley, Cheshire

SIR,-Lady Brook, president of the Brook Advisory Centres, says (12 August, p 499): "BAC are primarily concerned with helping young people to accept the responsibility of contraception and this is done within the context of counselling." I should have thought that the primary concern should be to help the young people of today to understand the place of the mechanics of sex in the much wider context of sexuality as a whole.

Dr Fay Hutchinson and her colleagues, also of the Brook Advisory Centres, say (p 499): "A girl under 16 cannot have a termination 